\title{
Front Matter: Volume 7332
}

, "Front Matter: Volume 7332," Proc. SPIE 7332, Unmanned Systems Technology XI, 733201 (28 May 2009); doi: 10.1117/12.832260

SPIE Event: SPIE Defense, Security, and Sensing, 2009, Orlando, Florida, United SPIE. States 


\section{PROCEEDINGS OF SPIE}

\section{Unmanned Systems Technology XI}

Grant R. Gerhart

Douglas W. Gage

Charles M. Shoemaker

Editors

14-17 April 2009

Orlando, Florida, United States

Sponsored and Published by

SPIE

Volume 7332 
The papers included in this volume were part of the technical conference cited on the cover and title page. Papers were selected and subject to review by the editors and conference program committee. Some conference presentations may not be available for publication. The papers published in these proceedings reflect the work and thoughts of the authors and are published herein as submitted. The publisher is not responsible for the validity of the information or for any outcomes resulting from reliance thereon.

Please use the following format to cite material from this book:

Author(s), "Title of Paper," in Unmanned Systems Technology XI, edited by Grant R. Gerhart, Douglas W. Gage, Charles M. Shoemaker, Proceedings of SPIE Vol. 7332 (SPIE, Bellingham, WA, 2009) Article CID Number.

ISSN 0277-786X

ISBN 9780819475985

Published by

SPIE

P.O. Box 10, Bellingham, Washington $98227-0010$ USA

Telephone +1 3606763290 (Pacific Time) · Fax +1 3606471445

SPIE.org

Copyright (C) 2009, Society of Photo-Optical Instrumentation Engineers

Copying of material in this book for internal or personal use, or for the internal or personal use of specific clients, beyond the fair use provisions granted by the U.S. Copyright Law is authorized by SPIE subject to payment of copying fees. The Transactional Reporting Service base fee for this volume is $\$ 18.00$ per article (or portion thereof), which should be paid directly to the Copyright Clearance Center (CCC), 222 Rosewood Drive, Danvers, MA 01923. Payment may also be made electronically through CCC Online at copyright.com. Other copying for republication, resale, advertising or promotion, or any form of systematic or multiple reproduction of any material in this book is prohibited except with permission in writing from the publisher. The CCC fee code is 0277-786X/09/\$18.00.

Printed in the United States of America.

Publication of record for individual papers is online in the SPIE Digital Library.

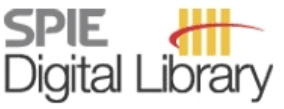

SPIEDigitalLibrary.org

Paper Numbering: Proceedings of SPIE follow an e-First publication model, with papers published first online and then in print and on CD-ROM. Papers are published as they are submitted and meet publication criteria. A unique, consistent, permanent citation identifier (CID) number is assigned to each article at the time of the first publication. Utilization of CIDs allows articles to be fully citable as soon they are published online, and connects the same identifier to all online, print, and electronic versions of the publication. SPIE uses a six-digit CID article numbering system in which:

- The first four digits correspond to the SPIE volume number.

- The last two digits indicate publication order within the volume using a Base 36 numbering system employing both numerals and letters. These two-number sets start with 00, 01, 02, 03, 04 , 05, 06, 07, 08, 09, OA, OB ... 0Z, followed by 10-1Z, 20-2Z, etc.

The CID number appears on each page of the manuscript. The complete citation is used on the first page, and an abbreviated version on subsequent pages. Numbers in the index correspond to the last two digits of the six-digit CID number. 


\section{Contents}

ix Conference Committee

SESSION 1 SELF-ORGANIZING, COLLABORATIVE, UNMANNED ISR ROBOTIC TEAMS I: JOINT SESSION WITH CONFERENCE 7350

733202 Fusion of inertial, optical flow, and airspeed measurements for UAV navigation in GPSdenied environments [7332-01]

A. Soloviev, Univ. of Florida (United States); A. J. Rutkowski, Air Force Research Lab. (United States)

733203 Wideband lasers on manned/unmanned platforms under poly-environment [7332-02] L. Shah, CREOL, The College of Optics and Photonics, Univ. of Central Florida (United States); J. Zeller, Naval Undersea Warfare Ctr. (United States); M. Richardson, CREOL, The College of Optics and Photonics, Univ. of Central Florida (United States); M. L. Talbert, Air Force Research Lab. (United States); G. S. Seetharaman, Air Force Institute of Technology (United States); T. Manzur, Naval Undersea Warfare Ctr. (United States)

SESSION 2 SELF-ORGANIZING, COLLABORATIVE, UNMANNED ISR ROBOTIC TEAMS II: JOINT SESSION WITH CONFERENCE 7350

733204 Integrated long-range UAV/UGV collaborative target tracking [7332-03]

M. B. Moseley, iRobot Corp. (United States); B. P. Grocholsky, Carnegie Mellon Univ. (United States); C. Cheung, iRobot Corp. (United States); S. Singh, Carnegie Mellon Univ. (United States)

733205 PRISTA UAVs: from troop companion to troop replacement [7332-04]

J. Maynell, Lite Machines Corp. (United States)

733207 Determining the position of runways from UAV video [7332-06]

R. Warren, A. Fischer, 21 st Century Systems, Inc. (United States)

733208 Complexity of robotic sensor networks [7332-07]

A. Mustapha, H. Singh, A. M. Dixit, K. Saab, Wayne State Univ. (United States); G. R. Gerhart, U.S. Army Tank-Automotive Research, Development and Engineering Ctr. (United States)

733209 Developing a UAV-based rapid mapping system for emergency response [7332-08] K. Choi, I. Lee, J. Hong, T. Oh, The Univ. of Seoul (Korea, Republic of); S. W. Shin, Electronics and Telecommunications Research Institute (Korea, Republic of)

\section{SESSION 3 SPECIAL TOPICS I: JOINT SESSION WITH CONFERENCE 7350}

7332 OA The DARPA LANdroids program [7332-09]

M. McClure, Defense Advanced Research Projects Agency (United States); D. R. Corbett, Schafer, Inc. (United States); D. W. Gage, XPM Technologies (United States) 
7332 OB Biologically inspired collision avoidance system for unmanned vehicles [7332-10]

F. E. Ortiz, EM Photonics, Inc. (United States); B. Graham, Univ. of Delaware (United States);

K. Spagnoli, E. J. Kelmelis, EM Photonics, Inc. (United States)

7332 OE Implementation of a piezoelectrically actuated self-contained quadruped robot [7332-13] T. Ho, S. Lee, Konkuk Univ. (Korea, Republic of)

\section{SESSION 4 SPECIAL TOPICS II}

7332 OG Advancing manufacturing research through competitions [7332-72]

S. Balakirsky, R. Madhavan, National Institute of Standards and Technology (United States)

7332 ol Robust formation control of multi-robot systems subject to interconnection time-delays using minimum dynamic communication [7332-15]

J. Zhang, S. Jayasuriya, Texas A\&M Univ. (United States)

7332 0J Preliminary results in force-guided assembly for teams of heterogeneous robots [7332-16] J. Rojas, Vanderbilt Univ. (United States); R. A. Peters II, Vanderbilt Univ. (United States) and Universal Robotics (United States)

SESSION 5

UGV TELE-OPERATION

7332 OL Field experiments using SPEAR: a speech control system for UGVs [7332-18]

S. R. Chhatpar, C. Blanco, Think-A-Move, Ltd. (United States); J. Czerniak, O. Hoffman, iRobot Corp. (United States); A. Juneja, T. Pruthi, Think-A-Move, Ltd. (United States); D. Liu, iRobot Corp. (United States); R. Karlsen, U.S. Army Tank-Automotive Research, Development and Engineering Ctr. (United States); J. Brown, Think-A-Move, Ltd. (United States)

7332 ON Flat panel 3D display for unmanned ground vehicles [7332-20]

J. L. Pezzaniti, R. Edmondson, J. Vaden, B. Hyatt, D. B. Chenault, Polaris Sensor Technologies, Inc. (United States); J. L. Tchon, T. J. Barnidge, Rockwell Collins (United States); B. Pettijohn, Army Research Lab. (United States)

733200 Improved situational awareness and mission performance for explosive ordnance disposal robots [7332-21]

K. Massey, Chatten Associates, Inc. (United States); J. Sapp, E. Tsui, U.S. Army Armament Research Development and Engineering Ctr. (United States)

7332 OP FOCU:S-future operator control unit: soldier [7332-22]

B. J. O'Brien, C. Karan, S. H. Young, Army Research Lab. (United States)

SESSION 6 INTELLIGENT BEHAVIORS

7332 OS Adaptable formations utilizing heterogeneous unmanned systems [7332-25] L. E. Barnes, The Univ. of Texas at Arlington (United States); R. Garcia, M. Fields, Army Research Lab. (United States); K. Valavanis, Univ. of Denver (United States)

7332 OU Road surveillance using a team of small UAVs [7332-27]

D. Kingston, Air Force Research Lab. (United States) 
$73320 \mathrm{~V}$ Discrete event command and control for networked teams with multiple missions [7332-28] F. L. Lewis, The Univ. of Texas at Arlington (United States); G. Hudas, U.S. Army TankAutomotive Research, Development and Engineering Ctr. (United States); C. K. Pang, The Univ. of Texas at Arlington (United States) and National Univ. of Singapore (Singapore); M. B. Middleton, C. McMurrough, The Univ. of Texas at Arlington (United States)

7332 OW Sequential learning for robot vision terrain classification [7332-29]

G. Witus, Turing Associates, Inc. (United States); R. Karlsen, U.S. Army Tank-Automotive Research, Development and Engineering Ctr. (United States); S. Hunt, Turing Associates, Inc. (United States)

7332 OY Neural network control of nonholonomic robot formations using limited communication with reliability assessment [7332-31]

T. Dierks, S. Jagannathan, Missouri Univ. of Science and Technology (United States)

$73320 Z$ Toward cognitive robotics [7332-70]

J. E. Laird, Univ. of Michigan (United States)

\section{SESSION 7 PERCEPTION}

733210 Stereo-vision-based terrain mapping for off-road autonomous navigation [7332-32]

A. L. Rankin, A. Huertas, L. H. Matthies, Jet Propulsion Lab. (United States)

733211 Using a laser range finder mounted on a MicroVision robot to estimate environmental parameters [7332-33]

D. Fehr, N. Papanikolopoulos, Univ. of Minnesota (United States)

733212 Characterization of the Hokuyo URG-04LX laser rangefinder for mobile robot obstacle negotiation [7332-34]

Y. Okubo, Univ. of Michigan (United States); C. Ye, Univ. of Arkansas at Little Rock (United States); J. Borenstein, Univ. of Michigan (United States)

733214 Tessellated structure from motion for midrange perception and tactical planning [7332-36] M. Shim, S. Yilma, General Dynamics Robotic Systems (United States)

733215 Detecting and tracking humans using a man-portable robot [7332-37]

D. Baran, N. Fung, S. Ho, J. Sherman, Army Research Lab. (United States)

733216 A stereo camera system for autonomous maritime navigation (AMN) vehicles [7332-38] W. Zhang, Technology Solutions Inc. (United States); P. Zhuang, L. Elkins, R. Simon, D. Gore, J. Cogar, Spatial Integrated Systems, Inc. (United States); K. Hildebrand, S. Crawford, Technology Solutions Inc. (United States); J. Fuller, Marshall Univ. (United States)

733217 Detection of moving targets from a moving ground platform [7332-39]

T. B. Sebastian, C. M. Wynnyk, P. H. TU, GE Global Research (United States); S. B. Barnes, Lockheed Martin Missiles and Fire Control (United States) 
733218 Increasing agility in unmanned ground vehicles using variable internal mass and inertial properties [7332-40]

C. Nie, S. C. Van Dooren, J. Shah, M. Spenko, Illinois Institute of Technology (United States)

733219 Stereo vision and laser odometry for autonomous helicopters in GPS-denied indoor environments [7332-41]

M. Achtelik, Technische Univ. München (Germany); A. Bachrach, R. He, S. Prentice, N. Roy, Massachusetts Institute of Technology (United States)

73321 A Test results of autonomous behaviors for urban environment exploration [7332-42]

G. Ahuja, D. Fellars, G. Kogut, E. Pacis Rius, B. Sights, H. R. Everett, Space and Naval Warfare Systems Ctr., Pacific (United States)

7332 1B Toward a generic UGV autopilot [7332-43]

K. L. Moore, M. Whitehorn, A. J. Weinstein, J. Xia, Colorado School of Mines (United States)

7332 1C An interactive physics-based unmanned ground vehicle simulator leveraging open source gaming technology: progress in the development and application of the virtual autonomous navigation environment (VANE) desktop [7332-44]

M. M. Rohde, J. Crawford, M. Toschlog, Quantum Signal LLC (United States);

K. D. Iagnemma, G. Kewlani, Massachusetts Institute of Technology (United States);

C. L. Cummins, R. A. Jones, D. A. Horner, U.S. Army Engineer Research and Development Ctr. (United States)

7332 IE Tracked robot controllers for climbing obstacles autonomously [7332-46]

I. Vincent, Defence R\&D Canada (Canada)

7332 iF Evaluation of terrain parameter estimation using a stochastic terrain model [7332-47]

D. A. Dumond, L. E. Ray, E. Trautmann, Dartmouth College (United States)

\section{SESSION 9 GOVERNMENT SESSION}

$733211 \quad$ Land, sea, and air unmanned systems research and development at SPAWAR Systems Center Pacific [7332-50]

H. G. Nguyen, R. Laird, G. Kogut, J. Andrews, B. Fletcher, T. Webber, R. Arrieta, H. R. Everett, Space and Naval Warfare Systems Ctr., Pacific (United States)

7332 i $\quad$ Evolving U.S. Department of Defense (DoD) unmanned systems research, development, test, acquisition, and evaluation (RDTA\&E) [7332-51]

R. T. Laird, Space and Naval Warfare Systems Ctr., Pacific (United States)

7332 IK TARDEC's Intelligent Ground Systems overview [7332-52]

J. F. Jaster, U.S. Army Tank-Automotive Research, Development and Engineering Ctr. (United States); 
7332 iN Joint collaborative technology experiment [7332-55]

M. Wills, D. Ciccimaro, S. Yee, Space and Naval Warfare Systems Ctr., Pacific (United States);

T. Denewiler, Science Applications International Corp. (United States); N. Stroumtsos,

NAVMAR Applied Sciences Corp. (United States); J. Messamore, R. Brown, L-3

Communications-MPRI (United States) and Air Force Research Lab. (United States);

B. Skibba, Air Force Research Lab. (United States); D. Clapp, J. Wit, Wintec, Inc. (United

States) and Air Force Research Lab. (United States); R. J. Shirts, U.S. Army Aviation and Missile

Research, Development and Engineering Ctr. (United States); G. N. Dion, Science

Applications International Corp. (United States) and U.S. Army Aviation and Missile Research, Development and Engineering Ctr. (United States); G. S. Anselmo, Science Applications International Corp. (United States)

733210 A vision-based robotic follower vehicle [7332-74]

J. L. Giesbrecht, Defence Research and Development Canada (Canada); H. K. Goi, Univ. of Toronto (Canada); T. D. Barfoot, Univ. of Toronto Institute for Aerospace Studies

(Canada); B. A. Francis, Univ. of Toronto (Canada)

\section{SESSION 10 MOBILE MANIPULATORS}

$73321 Q \quad 3 D$ visualization for improved manipulation and mobility in EOD and combat engineering applications [7332-57]

J. Alberts, J. Edwards, J. Johnston, J. Ferrin, Autonomous Solutions, Inc. (United States)

7332 IR OzBot and haptics: remote surveillance to physical presence [7332-58]

J. Mullins, M. Fielding, S. Nahavandi, Deakin Univ. (Australia)

7332 is Laser-assisted real-time and scaled telerobotic control of a manipulator for defense and security applications [7332-59]

E. Veras, K. Khokar, R. Alqasemi, R. Dubey, Univ. of South Florida (United States)

\section{SESSION 11 SMALL ROBOTS}

7332 iT Stingray: high-speed control of small UGVs in urban terrain [7332-60]

B. Yamauchi, iRobot Corp. (United States); K. Massey, Chatten Associates, Inc. (United States)

$73321 \mathrm{U}$ Implementation of small robot autonomy in an integrated environment [7332-61]

B. J. O'Brien, L. Sadler, Army Research Lab. (United States)

$73321 \mathrm{~V}$ Vision-based effective dispersion of miniature robots by using local sensing [7332-62]

H. J. Min, N. Papanikolopoulos, Univ. of Minnesota (United States)

7332 1X Ten-kilogram vehicle autonomous operations [7332-64]

J. R. Rogers, C. Korpela, K. Quigley, U.S. Military Academy (United States)

7332 1Y Inexpensive robot for remote detection of UXO [7332-73]

J. Galloway, D. R. Wilcox, Southern Polytechnic State Univ. (United States) 
733212 On software implementation of reliability of unmanned ground vehicles [7332-65]

A. M. Dixit, K. Saab, H. Singh, A. Mustapha, Wayne State Univ. (United States); G. R. Gerhart, U.S. Army Tank-Automotive Research, Development and Engineering Ctr. (United States)

733221 UGV application modeling and sensor simulation using a rapid prototyping testbed environment [7332-67]

J. Falasco, Crane Wireless Monitoring Solutions (United States); S. O'Leary, Mobile Robots Inc. (United States)

733222 Comparison of real-time performance of Kalman filter-based slam methods for unmanned ground vehicle (UGV) navigation [7332-69]

H. Temeltaş, D. Kavak, ìstanbul Technical Univ. (Turkey)

Author Index 


\title{
Conference Committee
}

\author{
Symposium Chair
}

Ray O. Johnson, Lockheed Martin Corporation (United States)

Symposium Cochair

Michael T. Eismann, Air Force Research Laboratory (United States)

Conference Chairs

Grant R. Gerhart, U.S. Army Tank-Automotive Research, Development and Engineering Center (United States)

Douglas W. Gage, XPM Technologies (United States)

Charles M. Shoemaker, General Dynamics Robotic Systems (United States)

Program Committee

Johann Borenstein, University of Michigan (United States)

Jonathan A. Bornstein, Army Research Laboratory (United States)

Bruce L. Digney, Defence Research and Development Canada (Canada)

Rajiv V. Dubey, University of South Florida (United States)

Hobart R. Everett, Space and Naval Warfare Systems Center, Pacific (United States)

Scott Fish, The University of Texas at Austin (United States)

David J. Gorsich, U.S. Army Tank-Automotive Research, Development and Engineering Center (United States)

Karl D. lagnemma, Massachusetts Institute of Technology (United States)

Jeffrey J. Jaczkowski, U.S. Army Tank-Automotive and Armaments Command (United States)

Gene A. Klager, U.S. Army Night Vision \& Electronic Sensors Directorate (United States)

Andreas F. Koschan, The University of Tennessee (United States)

James H. Lever, U.S. Army Corps of Engineers (United States)

Frank L. Lewis, The University of Texas at Arlington (United States)

Larry H. Matthies, Jet Propulsion Laboratory (United States)

Elena R. Messina, National Institute of Standards and Technology (United States)

Kevin L. Moore, Colorado School of Mines (United States)

Robin R. Murphy, University of South Florida (United States) 
James L. Overholt, U.S. Army Tank-Automotive Research, Development and Engineering Center (United States)

Marc Raibert, Boston Dynamics (United States)

Klaus-Juergen Schilling, Universität Würzburg (Germany)

Nahid N. Sidki, Science Applications International Corporation (United States)

Harpreet Singh, Wayne State University (United States)

Magnus S. Snorrason, Charles River Analytics, Inc. (United States)

Anthony Stentz, Carnegie Mellon University (United States)

David L. Stone, Mechatron Consulting (United States)

Venkataraman Sundareswaran, Teledyne Scientific Company (United States)

Brian H. Wilcox, Jet Propulsion Laboratory (United States)

Gary Witus, Turing Associates, Inc. (United States)

Brian M. Yamauchi, iRobot Corporation (United States)

\section{Session Chairs}

1 Self-Organizing, Collaborative, Unmanned ISR Robotic Teams I: Joint Session with Conference 7350

George Vachtsevanos, Georgia Institute of Technology (United States) Nahid N. Sidki, Science Applications International Corporation (United States)

Venkataraman Sundareswaran, Teledyne Scientific Company (United States)

2 Self-Organizing, Collaborative, Unmanned ISR Robotic Teams II: Joint Session with Conference 7350

Nahid N. Sidki, Science Applications International Corporation (United States)

Venkataraman Sundareswaran, Teledyne Scientific Company (United States)

3 Special Topics I: Joint Session with Conference 7350

Douglas W. Gage, XPM Technologies (United States)

Charles M. Shoemaker, General Dynamics Robotic Systems (United States)

4 Special Topics II

Douglas W. Gage, XPM Technologies (United States)

Charles M. Shoemaker, General Dynamics Robotic Systems (United States)

5 UGV Tele-Operation

Gary Witus, Turing Associates, Inc. (United States) 
Intelligent Behaviors

Frank L. Lewis, The University of Texas at Arlington (United States)

Gregory R. Hudas, U.S. Army Tank-Automotive Research, Development and Engineering Center (United States)

7 Perception

Magnus S. Snorrason, Charles River Analytics, Inc. (United States)

Larry H. Matthies, Jet Propulsion Laboratory (United States)

8 Mobility and Navigation

Karl D. lagnemma, Massachusetts Institute of Technology (United States)

Mel W. Torrie, Autonomous Solutions, Inc. (United States)

9 Government Session

Jonathan A. Bornstein, Army Research Laboratory (United States)

Jeffrey F. Jaster, U.S. Army Tank-Automotive Research, Development and Engineering Center (United States)

James L. Overholt, U.S. Army Tank-Automotive Research, Development and Engineering Center (United States)

10 Mobile Manipulators

Brian M. Yamauchi, iRobot Corporation (United States)

Robert E. Karlsen, U.S. Army Tank-Automotive Research, Development and Engineering Center (United States)

11 Small Robots

Stuart H. Young, Army Research Laboratory (United States) 
Downloaded From: https://www.spiedigitallibrary.org/conference-proceedings-of-spie on 26 Apr 2023

Terms of Use: https://www.spiedigitallibrary.org/terms-of-use 